\title{
Penser la didactique du français à travers des concepts de la didactique du FLES
}

Designing the teaching of French through concepts of teaching French as a foreign and second language

\section{Marie-Armelle Camussi-Ni}

\section{(2) OpenEdition}

\section{Journals}

Édition électronique

URL : https://journals.openedition.org/pratiques/9845

DOI : $10.4000 /$ pratiques. 9845

ISSN : 2425-2042

Éditeur

Centre de recherche sur les médiations (CREM)

Référence électronique

Marie-Armelle Camussi-Ni, « Penser la didactique du français à travers des concepts de la didactique du FLES », Pratiques [En ligne], 189-190 | 2021, mis en ligne le, consulté le 23 juillet 2021. URL : http:// journals.openedition.org/pratiques/9845; DOI : https://doi.org/10.4000/pratiques.9845

Ce document a été généré automatiquement le 23 juillet 2021.

(c) Tous droits réservés 


\title{
Penser la didactique du français à travers des concepts de la didactique du FLES
}

\author{
Designing the teaching of French through concepts of teaching French as a \\ foreign and second language \\ Marie-Armelle Camussi-Ni
}

\section{Introduction}

1 Le constat de l'utilité des démarches inclusives destinées aux élèves de Français langue étrangère et seconde (FLES) pour les élèves de Français langue première ${ }^{1}$ (FLP) en difficulté scolaire est généralement partagé, sans pour autant que ne soient forcément élucidés les concepts pertinents pour ces deux disciplines, dont il convient de souligner qu'elles sont bien distinctes. Nous nous proposons ici d'explorer quelques concepts centraux de la didactique du FLES, dans sa composante d'étude de la langue, susceptibles d'être exploités ou, tout au moins, davantage mis en valeur en didactique $\mathrm{du}$ français langue première et tout particulièrement dans une didactique du français qui se donne pour objectif la lutte contre les inégalités sociales au sein du système scolaire. .

2 Paradoxalement, ces concepts sont peut-être à chercher dans ce qui semble au fondement même de la différence entre la didactique du FLES et celle du FLP : la prise en compte du fait que l'usage de la langue enseignée n'est pas partagé par les apprenant·es FLES. L'enseignant de FLES est contraint, par la spécificité même de son public, de développer une didactique qui ne présuppose pas la pratique de la langue enseignée. Cette posture nécessaire de l'enseignant conduit à une vigilance toujours en éveil pour distinguer usage et système de la langue et aboutit à plusieurs principes ou démarches concomitantes qui tous peuvent avoir de l'intérêt pour l'élève francophone. 
Ce regard porté sur la didactique du FLES peut mettre en lumière un implicite selon lequel les élèves francophones disposeraient d'un usage de la langue partagé et conduire à le réexaminer. En effet, un faisceau d'études de didactique du français concourt à interroger de façon précise les acquis des élèves et à dresser, en définitive, un panorama qui remet nettement en cause cet implicite. Dès lors, peut se manifester un intérêt nouveau pour les concepts et démarches de la didactique du FLES dans l'enseignement du français.

\section{De l'usage partagé de la langue}

4 La spécificité de la didactique de la langue en FLS implique la prise en compte de l'usage explicitement non partagé par les apprenantes de la langue enseignée. Le public FLES contraint en effet l'enseignante à développer une didactique qui ne s'appuie pas sur des compétences basées sur l'usage des apprenant-es. La réflexion visant à établir la distinction entre l'usage et le système de la langue est donc particulièrement active en didactique du FLES et peut conduire à favoriser l'observation raisonnée de la langue.

De fait, les questions s'appuyant sur l'usage de la langue, fréquentes en FLP, sont insolubles pour les apprenant-es FLES et sont susceptibles de conduire leur enseignante dans une impasse. Pour en donner quelques exemples, un exercice qui demande de fournir le féminin d'adjectifs présentés au masculin ne peut pas convenir pour instituer une réflexion sur la morphologie flexionnelle auprès d'élèves de FLES, mais seulement correspondre à la restitution d'une mémorisation. De même, l'exercice suivant extrait d'un manuel de $2^{\text {nde }}$ ou tout exercice de ce type ne sera pas réalisé de la même façon par des élèves allophones que par des élèves francophones :

Associez chaque numéro à un des pronoms relatifs suivants : lequel, dont, que, qu', qui :

Ombrée par les montagnes (1)... la surplombent, cette bourgade encadrait très bien sa personne. À portrait sombre, cadre sombre. La baronne de Ferjol, âgée d'un peu plus de quarante ans, était une grande brune maigre (2).... la maigreur semblait éclairée en dessous d'un feu secret, brûlant comme la cendre, dans la moelle de ses os... Belle, - les femmes disaient qu'elle l'avait été autrefois mais agréable, non! Ajoutaient-elles avec le plaisir (3)... leur causent d'ordinaire, ces atténuations. Sa beauté (4) ... n'avait été désagréable, du reste, aux autres femmes, que parce qu'elle avait été écrasante, elle l'avait enterrée avec l'homme (5) ... elle avait éperdument aimé... (Beltrando, 2019, p. 423)

6 Les francophones mobilisent quasi-intuitivement les pronoms relatifs pour faire un énoncé grammatical, sans convoquer d'analyse - et ce, quand bien même leur intuition ne correspondrait pas à la norme. Les apprenantes allophones sont bien incapables d'un tel appui sur l'usage et il convient de leur proposer une procédure pour réaliser l'exercice : leur montrer, par exemple, l'absence de sujet ou de complément d'objet du verbe de la relative, ce qui peut demander l'apport de connaissances sur la transitivité de certains verbes. Au total, une démarche loin d'être aussi fluide que l'appui sur l'usage mais incontournable.

7 De même, la correction des productions de l'apprenant allophone est un bon exemple de la vigilance qui s'impose à l'enseignante de FLES : s'il ne veut pas produire de ruptures intempestives dans la communication, l'enseignantee est amenée à trier entre reprises utiles et superflues. La distinction entre usage et système est une entrée efficace puisqu'elle lui permet de sélectionner des reprises pertinentes qui pourront être appliquées à toutes sortes d'autres énoncés produits par l'apprenant et de négliger 
d'autres erreurs liées à l'usage et donc moins susceptibles d'être reproduites. Pour en donner un exemple, si l'enseignant·e reconnait dans un énoncé erroné comme ${ }^{*}{ }_{2} i l$ lui discute une confusion entre le pronom personnel conjoint lui et son homophone, le pronom personnel disjoint lui (il discute avec lui), confusion dont peut découler toute une série d'erreurs, il sera amené à expliciter sa source à l'aide de pronoms non homophones (me/moi; leur/eux : il leur parle/il discute avec eux). À l'inverse, il gagnera à ne pas s'attarder sur une erreur comme ce sera mieux si vous buvez un café moins susceptible d'être reproduite et qui relève plutôt de l'usage.

Aussi l'enseignante de FLES n'a pas le choix : ileelle doit exercer sa vigilance sur les démarches convoquées par les exercices et vérifier qu'elles sont bien adaptées à ses apprenantes. Il lui est donc nécessaire de distinguer nettement usage et système dans l'apprentissage proposé, ce qui est sans doute moins flagrant dans la démarche de l'enseignante de Français langue première.

9 En effet, dans cet enseignement perdure un implicite selon lequel il est possible de s'appuyer sur un usage commun aux élèves francophones et à leurs enseignant·es qui innerve certains exercices des manuels de français mais aussi les conceptions des enseignantes qui s'appuient souvent de façon inconditionnelle sur cet usage commun. En témoigne, à titre d'exemple, ce type de consignes relevées aussi bien dans des manuels du premier degré que dans des manuels de lycée :

À quel nom propre renvoient les pronoms il ? Qui ce nom propre désigne-t-il ? Relève les déterminants qui renvoient au même personnage? (Huchet \& Bastier, 2019 [2017], p. 24)

Réécrivez en mettant au passé simple les verbes à l'imparfait. Attention à la concordance des temps. (Damas, 2019, p. 37)

Plus largement, le fait de s'adresser à un public francophone induit implicitement une exigence maximale vis-à-vis des réalisations en langue française. C'est ce que relève D. Cogis (2001), dans le domaine de l'orthographe : elle déplore une conception sousjacente à l'évaluation de l'orthographe dans laquelle la production de l'élève est vue comme déficitaire par rapport à un savoir expert posé comme horizon d'attente dès le début de l'apprentissage de l'écriture.

11 Cette posture exigeante qui convoque un déjà-là de la langue est à l'œuvre, d'une façon ou d'une autre, dans tous les domaines de la langue, compréhension fine des énoncés, syntaxe, lexique..., ce qui peut justifier alors une moindre attention de l'enseignant.e aux notions pourtant cruciales de progression et de prérequis dans les apprentissages.

De plus l'usage de la langue qui est figuré comme très achevé chez les élèves recouvre une conception normée, souvent éloignée de leur réalité mais aussi de celle de la langue qui admet, outre la norme, des variantes.

13 J. Boutet (2002) témoigne des impasses pédagogiques auxquelles conduit le présupposé d'un usage partagé quand elle rend compte d'un échange entre une enseignante débutante et sa classe. Dans cet échange, l'enseignante «n'entend pas» la solution proposée à plusieurs reprises par un élève et redite par la classe «cette voiture l'appartient ». Le malaise de l'enseignante témoigne de la déstabilisation provoquée par un impensé en classe de français langue première : le non-partage, ponctuel, de l'usage normé de la langue.

Or, souligne E. Guérin $(2010 ; 2011)$, cet usage partagé de la langue sur lequel se fonde l'enseignante est souvent un mythe qui ne correspond pas à la réalité des variations de la langue. Aussi peut-il conduire l'enseignant-e à fustiger chez l'élève des énoncés qu'il 
est susceptible de produire lui-même, comme des interrogatives sans inversion, des accords non réalisés de participes passés à l'oral, des négations tronquées, un choix inadéquat du pronom relatif, etc.

\section{Des études mettant en cause une vision idéalisée de l'appréhension de la langue en Français langue première}

Les chercheures en didactique du français ne sont pas dupes de ce décalage entre usage représenté et usage réel de la langue de l'élève francophone. Tout un faisceau d'études en didactique du français ou en psycholinguistique déstabilise, d'une façon ou d'une autre, l'implicite d'une langue partagée par les élèves.

de recherches actives en didactique du français examine ainsi la corrélation entre difficultés grammaticales ou orthographiques et fréquentation effective des faits de langue ou de lexique peu fréquents.

17 Par exemple, D. Cogis (2004) montre, à partir des évaluations nationales des élèves de $6^{e}$ des années 1990-2002, que l'accord de certains adjectifs est moins bien maitrisé que d'autres et ce, de façon systématique, quantifiable statistiquement : $49,8 \%$ de réussite pour les adjectifs moins bien maitrisés et $79,6 \%$ pour les mieux maitrisés. Ainsi est-il frappant de relever que, quelles que soient les années de passation, les adjectifs trempées et glissées prennent les 5 premières places, indifférentes et majestueuses les 4 suivantes, alors qu'impressionnées et enthousiastes se situent entre le $11^{\mathrm{e}}$ et le $14^{\mathrm{e}}$ rang. En définitive, les élèves doutent de la catégorie de certains adjectifs comme malpoli dès lors qu'ils sont moins fréquents que d'autres comme pauvre, petit. D. Cogis (2004) commente :

Là où l'expert semble produire des marques d'accord sans distinction, eux se montrent très dépendants des circonstances, sans parler de leurs capacités de traitement et de contrôle. (Cogis, 2004, p. 76)

18 Dans leur appréhension de la langue, les élèves de $6^{\mathrm{e}}$ sont donc loin d'une acquisition complète de la classe des adjectifs et de leur accord, contrairement à ce que prévoient les Instructions Officielles en France ${ }^{3}$ mais surtout, ils n'ont pas une maitrise homogène de cette classe grammaticale.

Dans la même perspective, plusieurs études de didacticien.nes ou de psycholinguistes portant sur les acquisitions orthographiques ont éclairé, à l'aide de protocoles permettant d'établir des statistiques, le fait que les élèves ne traitent pas de façon équivalente tous les lexèmes de la langue, mettant en évidence l'influence de leur fréquentation et par conséquent de la mémoire dans les performances.

20 Ainsi, C. Totereau, P. Barrouillet et M. Fayol (1998) ont-ils montré que les erreurs dans le choix de la marque du pluriel sont dépendantes de la fréquence à laquelle le radical du mot a été présenté aux élèves en tant que nom ou en tant que verbe. Victimes de ce que ces auteurs nomment "l'effet d'homophonie verbo-nominale ", les élèves classent, en définitive, un mot dans une catégorie fixe quand ils l'ont majoritairement rencontré dans cette catégorie. Par exemple, ils écriront *des rêvent si rêve apparait majoritairement sous la forme ils rêvent. Les auteurs démontrent ainsi l'influence de la fréquentation d'occurrences pris dans leur globalité dans l'appréhension de la langue des élèves francophones. 
21 Suivant le même type d'hypothèse sur la mémorisation dans l'apprentissage, P. Largy, M.-P. Cousin et M. Fayol (2004) ont démontré, à l'aide d'une étude longitudinale contrôlée expérimentalement, en exposant directement des élèves à des exercices de lecture et de copie de noms rares au singulier ou au pluriel, l'influence de la forme rencontrée sur la forme produite ultérieurement. Ils constatent que la capacité à produire la flexion nominale juste dépend de la fréquence des formes fléchies : ainsi les noms plus souvent rencontrés au pluriel seront-ils davantage orthographiés au pluriel et vice versa.

22 L'influence de la fréquentation des formes est également au centre d'une recherche menée en 2006 par C. Brissaud, J.-P. Chevrot et P. Lefrançois. S'appuyant sur des recherches portant sur l'acquisition de la langue à l'oral qui ont montré que s'effectuait une mémorisation associative de séquences globalisées au détriment d'une mémorisation analytique (Bybee, 1985; Bybee \& McClelland, 2005), les auteurs explorent l'influence de la mémorisation de formes en $\mathrm{E}$ chez les élèves et démontrent que le choix d'orthographier une finale en $\mathrm{E}$ «er» ou "é» est dépendant de la fréquence à laquelle ils ont été exposés à l'une ou à l'autre forme de chaque verbe dans les manuels. L'orientation des verbes vers «er » ou vers "é » a statistiquement une influence sur le choix de la forme, qu'elle soit correcte ou erronée.

C. Brissaud, J.-P. Chevrot \& P. Lefrançois (2006, p. 75) l'analysent ainsi :

Des instances bases + flexions sont mémorisées par l'apprenti-scripteur. [...] Le scripteur mémorise des séquences d'unités orthographiques qu'une analyse linguistique considère comme indépendantes.

24 Ainsi ces études convergent-elles pour démontrer que l'usage dont bénéficient les élèves francophones ne leur permet pas d'accéder à une appréhension précise et complète de la langue française. Finalement, même s'ils ont un usage du français beaucoup plus diversifié que les allophones, les élèves francophones construisent, comme eux, une vision parcellaire et parfois faussée de la langue.

\section{Déconstruction et fossilisation}

Or, des éléments de ces études laissent à penser que les élèves francophones pourraient bénéficier, comme les élèves allophones, d'une déconstruction analytique d'une langue qu'ils perçoivent de manière trop indifférenciée.

26 Par exemple, l'étude de C. Totereau, P. Barrouillet et M. Fayol (1998) sur les "homophonies verbo-nominales", rend compte aussi de ce qui est rendu visible à l'apprenti-scripteur, c'est-à-dire le découpage en "mots ", notion dont on sait qu'elle n'a pas de réalité proprement linguistique ${ }^{4}$. On peut d'ailleurs remarquer que la scolarisation en France conforte l'effet de la fréquence de ces "homophonies verbonominales", dans la mesure où il est préconisé une mémorisation des «mots" sélectionnées en fonction de leur fréquence et classés dans des catégories noms, verbes, adjectifs ${ }^{5}$, ce qui renforce l'effet pervers de fréquence repéré. Ce dernier est d'autant plus fort que l'accent est mis sur le radical présenté seul - sans les morphèmes qui pourraient spécifier sa catégorie - donc sur le sens lexical. Ainsi ce phénomène d'association, qui est supputé être à l'œuvre dès l'acquisition à l'oral, pourrait être renforcé par l'apprentissage de l'écrit, alors qu'on pourrait imaginer que le passage à 
l'écrit soit le moment de la déconstruction, de la mise en distance, de la conceptualisation, comme le rappelle ici J.-L. Chiss (1997) :

Des ethnologues, comme Jack Goody, ont attiré l'attention - confirmée par une multitude de travaux - sur la part décisive qu'occupe l'écriture dans la codification des savoirs, au point de se demander si les mathématiques, la géographie, la grammaire auraient été concevables sans la scripturalité. Entendue au sens d'activité cognitive permettant l'abstraction, la conceptualisation, la généralisation, l'écriture est donc une manière de penser. frappant que les auteurs rapportent que l'effet de la mémorisation de formes au singulier ou au pluriel perdure davantage quand les formes ont été rencontrées avant la présentation de la règle (en $\mathrm{CP}$ ) qu'après (en $\mathrm{CE} 1$ ).

$\mathrm{Au} \mathrm{CP}$, les enfants accordent mieux au pluriel les noms qu'ils ont rencontrés ainsi fléchis et produisent moins bien au singulier les noms précédemment rencontrés au pluriel. De plus, le phénomène résiste à l'enseignement de la règle. [...] Alors que (l'impact) disparaît systématiquement dès décembre chez les ex-CE1, il ne disparaît qu'en mars de la deuxième année chez les ex-CP. [...] Il se pourrait que l'élève de 6-7 ans mémorise de manière plus stable et/ou plus durable l'enveloppe orthographique globale des mots que ne le ferait un enfant plus âgé. Il se pourrait aussi que les enfants plus âgés bénéficient, pour l'analyse et la mise en œuvre de la morphologie flexionnelle du nombre, de pratiques métalinguistiques plus étendues. (ibid., p. 51)

Cette remarque, qui demanderait à être prolongée par des expériences comme l'indiquent les auteurs, tend à démontrer qu'une analyse grammaticale limite dans le temps la perception erronée et inversement. Elle fait écho aux études déjà anciennes en didactique de la langue étrangère, sur la fossilisation d'une étape d'interlangue (Selinker, 1972, Selinker \& Lakshamanan, 1992) ${ }^{6}$. Ce concept d'interlangue peut être conçu comme une dynamique correspondant à une construction progressive de la langue cible dans laquelle les erreurs sont vues comme autant d'étapes provisoires. La fossilisation, quant à elle, marque l'arrêt de cette dynamique. Les apprenantes allophones construisent des grammaires provisoires à différentes étapes et leur confrontation à la langue cible doit permettre de dépasser ces étapes en les révisant. Or, il apparait que le processus peut se gripper, aboutissant alors à la fossilisation d'une étape d'interlangue. On peut définir celle-ci comme la fixation d'une conception erronée de la langue-cible qui va perturber durablement sa maitrise par l'apprenant. Un exemple, parmi d'autres: cette réalisation de la détermination chez une apprenante qui vit en France et pratique le français depuis plus de vingt ans : ${ }^{*} c e t$ l'exercice.

31 Si L. Selinker (1992) souligne une cause spécifique à l'apprentissage de la langue étrangère dans la fossilisation d'un état d'interlangue, l'effet du transfert de la langue 1 sur la langue cible, il indique que ce peut être aussi l'effet de "surgénéralisation de 
certaines règles » ou de stratégies d'apprentissage comme la simplification du système de la langue cible.

Ces causes moins évidentes de la fossilisation, qui ne se rattachent plus aux interférences de la langue première, peuvent nous conduire à interroger la pertinence de ce concept dans d'autres domaines que celui de la langue étrangère et pour ce qui nous concerne, dans celui du français langue première.

Le concept de fossilisation ne fait à priori pas partie de l'arsenal des didacticiens du français. Aussi n'a-t-il jamais été exploré, à notre connaissance, dans des études de didactique du français langue première. Pourtant, des remarques dans les études sur la persistance de représentations erronées de la langue chez des locuteurs francophones peuvent inciter à y voir un concept opérationnel pour l'enseignement du français langue première, susceptible de modifier les pratiques par prise de conscience des enjeux. Nous avons regroupé ici quelques exemples mais il serait sans doute judicieux d'explorer de façon plus systématique cette hypothèse.

34 Ainsi la présentation des «mots» en fonction de leur fréquence occulte-t-elle qu'un même radical peut construire différentes catégories morphologiques et on peut repérer une résistance à la recatégorisation chez certain'es élèves, renforcée certainement par leur représentation sémantique, mais qui ne cède pas devant une nouvelle analyse. C'est le cas pour l'élève de CE2 nommé Joan, dans cet échange métalinguistique avec son enseignante autour de l'énoncé Tu ne sucres pas le gâteau :

Joan : Je réécris mais là, y a ne et pas entre le sucre. Le sucre, c'est pas un verbe !

M. : Qu'est ce que c'est, sucre?

Joan : C'est un nom. Parce qu'en fait j'ai lu la phrase et j'ai vu qu'entre sucre il y a ne

et pas mais que sucre c'est pas un verbe.

M. : Pourquoi ?

Joan : Bah parce que c'est un nom ! (Camussi-Ni, Coatéval \& Folmer 2016)

L'aspect définitif de la dernière réplique de Joan montre que la catégorisation qu'il a opérée du radical sucre, est intangible dans le sens où elle résiste à la démonstration grammaticale qu'il réalise lui-même en encadrant sucre par les morphèmes ne et pas dont il sait qu'elle prouve l'appartenance à la catégorie verbale. L'exemple témoigne donc que l'étiquetage peut avoir plus de force que la preuve. Dès lors, la représentation de l'élève est bien fossilisée dans le sens où elle semble difficile à déconstruire.

Ce type de confusion peut perdurer comme en témoigne cet exemple extrait cette fois d'échanges métagraphiques en classe de $4^{\mathrm{e}}$ :

"Les Bleus sont allés en Russie, ils sont parties en avion " Je ne sais pas très bien pourquoi j'ai écrit [es] à parties, mais je suis presque sûr que c'est correct car on dit bien « une partie ». (Extrait du mémoire de Master II de P. Lecointre- Université Rennes 2)

37 La remarque de l'élève témoigne ici de la prédominance d'une forme mémorisée qui conduit à une absence de distinction entre un nom et un verbe, en l'occurrence homophones. La connaissance des mots se fige autour de formes enregistrées telles quelles dans une catégorie.

La résistance de certaines analyses erronées fondées sur des rapprochements sémantiques peut également interroger sur la possibilité d'une fossilisation des apprentissages chez certain.es élèves en français langue première. 
39 En effet, des chercheurs en didactique du français ont mis au jour des étapes dans l'apprentissage de l'orthographe. Ainsi, D.Cogis (2005) liste-t-elle une série de procédures dont la dernière serait la plus opérationnelle :

- les procédures de type phono/logographique transcrivent les sons ;

- les procédures de type morphosémantique s'appuient sur un raisonnement sémantique ;

- les procédures de type morphographique sur la catégorie du mot;

- les procédures de type morphosyntaxique sur les relations syntaxiques entre les mots.

Or, certains locuteurs semblent ne pas dépasser l'étape morphosémantique, opérant ce qui semble fort correspondre à une fossilisation d'une étape d'apprentissage. En témoignent ces quelques exemples d'une procédure morphosémantique qui s'est grippée :

«Les bleues sont allées en Russie » Bleu, c'est une couleur et donc, comme c'est féminin, ça prend un [e] à la fin. Et comme bleues, c'est féminin, j'accorde allées au féminin aussi.

«Ils sont partie en avion » Je me suis dit que ils, c'est une équipe de foot et donc, il fallait mettre un [e] parce que une équipe, c'est un sujet féminin. Mais c'est pas ça, si vous me demandez.

Élève de $4^{\mathrm{e}}$ (Extrait du mémoire de Master II de P. Lecointre Université Rennes 2)

«ses yeux verts voit ». [L'élève] passe par une analyse sémantique (« on parle du petit garçon ») qui l'amène à identifier le petit garçon comme sujet et donc à remplacer par il et à choisir la forme voit. Cette procédure de remplacement du sujet par un pronom ne semble donc pas faire obstacle à condition d'identifier correctement le sujet par une procédure syntaxique et non sémantique.

Élève de $3^{\mathrm{e}}$ (Le Levier, Brissaud \& Huard, 2018, p. 14)

«Le plafond s'effritent. » Parce que quand le plafond s'effrite, ça fait plein de petites miettes partout. Donc pluriel.

Adulte, 32 ans. (Cogis, 2001, p. 58)

D. Cogis (2001) pose d'ailleurs l'hypothèse que ce dernier cas serait dû à un « arrêt du développement des procédures ", ce qui correspond à la définition de la fossilisation dans l'apprentissage :

On peut alors tenter - à titre d'hypothèse de travail - une nouvelle approche des difficultés d'apprentissage en orthographe. Celles-ci seraient, sans doute pour une bonne part, à repérer dans un arrêt du développement des procédures: une procédure (ou un ensemble de procédures) se fige, car elle est jugée par l'apprenant comme suffisamment efficace. Il s'agit des procédures non retravaillées par la syntaxe, parfois les procédures phonographiques, mais, surtout, les procédures morphosémantiques. (Cogis, 2001, p. 58)

42 Le terme « fossilisation » est également employé par M.-L. Élalouf (2005) non plus pour décrire une étape de raisonnement mais un traitement sclérosé du métalangage. Dans le cadre d'une étude de l'évolution des explicitations métalinguistiques des élèves entre la $6^{\mathrm{e}}$ et la $1^{\mathrm{re}}$, M.-L. Élalouf décrit une catégorie d'élèves qui «mobilisent [...] des connaissances "fossilisées" en décalage avec les intuitions que traduisent leurs réponses » (ibid., p. 177).

43 Allant dans le même sens, P. Gourdet et M.-N. Roubaud (2016, p. 10) s'interrogent sur certaines réponses produites par les élèves dans une étude sur le repérage du verbe :

Ces réponses données dans un environnement scolaire (la classe), apparaissent souvent comme une "déclaration de règles", les élèves reprenant le discours déclaratif des maîtres d'une façon mécanique. [...] Nous avons l'impression d'avoir une explication très incantatoire et peu opérationnelle qui nous amène à poser cette question : cet élève est-il capable d'identifier un verbe et de l'utiliser? 

différencier fermement usage et système et à valoriser des démarches qui visent la déconstruction du système plutôt que des démarches qui valident des usages déjà acquis.

Seul David répugne à intégrer ces formes particulières dans le regroupement des déterminants; trois est par deux fois positionné par cet élève dans le rassemblement des adjectifs, ce qui peut s'expliquer par les importants savoirs déclaratifs de cet élève : trois est un adjectif numéral et cette représentation prend le pas sur tout autre raisonnement. (ibid., 2018, p. 161)

Dans un article de 1997 qu'elle consacre à ce sujet, A. Trévise analyse cette fossilisation du métadiscours qu'elle attribue à un emploi très prégnant du métalexique chez les jeunes apprenantes français interdisant toute émergence de la réflexion métalinguistique spontanée :

Cela est frappant lorsque l'on étudie des manuels, des discours d'enseignants, des interactions en classe, des verbalisations d'apprenantes de divers âges et niveaux, dans divers contextes. Les mises en métadiscours ne font manifestement, dans l'état actuel des choses du moins, pas vraiment sens. Les métatermes n'ont pas de réel pouvoir référentiel : on a alors l'impression que l'on est devant des mises en œuvre de métatermes et du discours métalinguistique qui ne sont pas bloquées par des contradictions flagrantes, comme le serait toute autre pratique langagière. Les contradictions ne sont manifestement pas des obstacles qui feraient surgir une conscience, comme c'est le cas «normalement» dans la communication disons « ordinaire ». L'incohérence ne semble en effet pas un obstacle qui fasse affleurer le doute, et la conscience, dans les verbalisations métalinguistiques des sujets, apprenantes et enseignants. L'absurde et le non-sens dominent encore trop souvent. (Trévisse, 1997, p. 48-50)

Aussi, peut-on s'interroger : certaines démarches d'apprentissage présenteraient-elles des risques de fossilisation de raisonnements inopérants chez les élèves francophones au même titre que l'apprentissage d'une langue étrangère ?

Au total il faudrait évaluer quantitativement l'importance du risque de fossilisation encouru par les élèves francophones dans leurs acquisitions aussi bien linguistiques que métalinguistiques. Cependant, l'intérêt suggéré ici de la transposition de ce concept propre à la didactique des langues étrangères à la didactique du FLP conforte l'idée de s'inspirer de la didactique du FLES pour la didactique du français langue première.

\section{Dès lors, quelles conséquences pour la didactique du français?}

La première conséquence de ces parallèles consiste à inciter les enseignants à 
49 Ne pas présupposer un usage partagé par tous de la langue de l'école permet ainsi d'éviter des situations d'apprentissage, somme toutes stériles, qui ne visent qu'à l'affichage d'un usage et non à son apprentissage pour favoriser des situations d'apprentissage qui, explicitant les formes, permettent d'en rendre l'apprentissage efficient.

Si des études comme celle de P. Largy, M.-P. Cousin \& M. Fayol (1998) conduisent à penser que les items non déconstruits en orthographe sont enregistrés tels quels dans la mémoire, conduisant à des formes de fossilisation, alors que l'introduction de l'analyse rend caduque cette démarche erronée, on peut dès lors se demander s'il ne serait pas nécessaire de procéder à une déconstruction parallèlement à la présentation des formes. Ou, à tout le moins, s'il ne faudrait pas veiller à présenter de façon systématique la variation des formes en nombre, en genre, en personne pour déjouer ce phénomène de fossilisation. Admettre que les élèves ne partagent pas forcément tous la langue de l'école peut ainsi conduire à favoriser des démarches qui leur proposent $\mathrm{du}$ corpus d'observation comme cela est pratiqué en FLES.

51 La didactique du FLES préconise la construction d'une grammaire implicite (Vigner, 2001) dans laquelle le système de la langue se construit par l'observation raisonnée de la langue, ce qui peut passer par l'utilisation de grands corpus qu'on apprend à manier dans le cadre de l'apprentissage de la langue (Boulton \& Tyne, 2014) ou de corpus restreints fabriqués, éventuellement à partir des premiers, pour favoriser l'induction (Camussi-Ni, Coatéval \& Thuilier, 2016). L'idée est de compenser le déficit de corpus de l'apprenant FLES en présentant un échantillon de corpus, rendant compte de façon économique d'un fait de langue. La didactique du FLES favorise ici une démarche de déconstruction des notions de la langue pour les rendre repérables et appréhendables. Ces corpus doivent permettent de comprendre la logique de la langue, sans passer par du métalangage. En effet, ne pas penser l'usage de la langue comme partagé par tous s'applique en FLES au métalangage dont l'usage peut sembler alourdir l'apprentissage, ce qui justifie d'une démarche de grammaire implicite. Or, la tendance de simplification du métalangage semble aujourd'hui un objectif partagé par la didactique du français. En témoignent par exemple, les Instructions officielles de français 2015 qui cherchent à limiter, au moins pour le cycle 2 et le cycle 3, les termes métalinguistiques (AvezardRoger \& Gourdet, 2017).

52 Ce principe de contournement du métalangage (au moins dans les premiers moments de l'apprentissage d'une notion), de l'appel au raisonnement plutôt qu'à la mémorisation est propre à inclure tous les élèves, dont ceux qui se découragent devant le métalangage grammatical. Il présente aussi l'avantage de contourner l'écueil d'un métalangage "fossilisé » qui fait obstacle au raisonnement des élèves comme le préconise M.-L. Élalouf (2005, p. 178) :

Compte tenu de l'obstacle que représentent les savoirs fossilisés, il semble utile d'exiger dans un premier temps des explications ou justifications qui ne font pas appel au métalangage, avant de proposer quelques termes correspondant à des besoins de désignation identifiés.

53 Aussi ces options didactiques du FLES peuvent-elles inspirer le développement de démarches de découverte euristique en français, à l'instar de celles déjà menées (David \& Renvoisé, 2010 ; Tisset, 2010 ; Brissaud \& Cogis, 2011 ; Chartrand, 2016 ; BeaumanoirSecq, 2018, pour en donner quelques exemples). 

vérifié par des études, il serait alors utile d'examiner les fausses représentations des élèves francophones pour les prévenir par l'enseignement, comme cela est proposé en didactique du FLES. Pour en donner un exemple, voici un mini-corpus qui vise à anticiper les risques liés à l'homophonie en FLES par la présentation des commutations de ces homophones :

- Elle fait un peu de baby-sitting avec une petite fille de 4 ans. C'est la petite fille de

la voisine.

- Ah, c'est cette petite fille-là ?

- Oui, elle la conduit à l'école et elle me conduit ensuite à la fac.

- Elle te conduit tous les jours?

- Non, quand même pas! (Camussi-Ni \& Coatéval, 2013)

Le bref échantillon de langue qui met en présence le déterminant la et le pronom complément la, homophones, offre par l'utilisation de paires minimales (elle la conduit/ elle me conduit, elle te conduit; la petite fille/une petite fille, cette petite fille) le moyen de les distinguer et par là, de distinguer le nom et le verbe. Ce type de corpus peut inspirer une démarche de découverte de la langue et d'anticipation des erreurs? ${ }^{7}$.

Enfin, nous l'avons vu, la reconnaissance d'un usage non partagé recouvre aussi la question sociolinguistique dans l'enseignement de la langue. Ce que nous apprennent les études en sociolinguistique et en psycholinguistique sur l'accueil de la langue étrangère à l'école (Laparra, 2003), c'est que l'intégration des élèves allophones est meilleure dans les collèges qui acceptent l'usage de la langue étrangère que dans ceux qui la prohibent. De fait, la prise en compte de la langue première de l'apprenant lui permet de se sentir reconnu dans son identité et d'éviter la diglossie, c'est-à-dire le sentiment que sa langue première est inférieure à la langue d'accueil. Aussi une démarche comme celle préconisée par N. Auger dans le DVD Comparons nos langues (2005) présente-t-elle l'intérêt d'introduire la langue étrangère dans les activités linguistiques de la classe, laissant la porte ouverte aux langues premières des élèves.

Ces réflexions très avancées en didactique du FLES sont susceptibles d'inspirer les postures des enseignantes en français langue première. Quel est l'impact psychologique chez l'élève dont on ignore l'usage? Quel est l'effet, même sur les bons élèves, d'analyses qui tournent à l'incohérence en niant l'usage réel des francophones ? C'est ce que se demande E. Guérin quand elle constate que des manuels cantonnent l'usage du passé composé à une langue orale ou sinon familière à l'écrit (Guérin, 2011, p. 66) construisant une langue proprement mythique.

58 La question des effets de l'incohérence de la représentation du français par rapport à l'usage réel des locuteurs est soulevée aussi par l'étude réalisée par C. Péret et J.P. Sautot (2018), qui démontre que l'emploi comme auxiliaire du verbe aller est occulté de façon très problématique dans l'enseignement fourni aux élèves pour laisser toute la place au futur simple pourtant beaucoup moins fréquent. Ce ne sont donc pas seulement des variantes périphériques qui seraient exclues dans l'enseignement du français mais aussi des éléments de la langue standard, comme le passé composé ou le futur périphrastique, au profit d'une langue écrite élaborée.

Mettre en doute l'idée implicite de l'usage partagé de la langue en didactique du français aboutit ainsi à une ouverture à tous les usages, à la non-imposition d'un usage normé de la langue comme le seul valable. Il n'est bien évidemment pas secondaire d'accueillir tous les usages en classe pour favoriser la justice sociale mais aussi pour ne 
pas occulter des usages qui, venant réinterroger implicitement l'analyse grammaticale, sont susceptibles de perturber la compréhension des élèves.

Si l'objectif d'enseignement est bien de mettre au jour le système de la langue et non d'entériner l'usage, il devient alors parfaitement légitime de prendre en compte les variantes du système du français dans l'enseignement, de la même façon qu'on peut comparer des langues en FLES. Ce qui n'empêche pas d'ailleurs l'enseignante de rattacher ces variantes à des contextes d'emplois et donc l'école de remplir son rôle normatif.

De fait, il semble intéressant d'explorer la piste de l'étude de variantes sociolinguistiques qui peuvent rendre compte du système à l'œuvre au même titre que l'usage normé de la langue et qui, en trouvant alors leur place dans les objets enseignés à l'école, légitimeraient leurs locuteurs, comme le propose déjà M. Laparra (2003). Par exemple, le refus de l'énoncé «Cette voiture l'appartient » relevé par J. Boutet (2002) témoigne du caractère non audible en classe d'une variante sociologique dont on pourrait facilement imaginer l'intégration dans l'analyse de la transitivité verbale et de ses variantes historiques ou polysémiques. Présenter quelques exemples de ces variations comme lui aider au lieu de l'aider, l'obéir au lieu de lui obéir, le souffrir au lieu d'en souffrir ${ }^{8}$ permet de relativiser l'erreur par rapport à la norme actuelle et de lui redonner sa juste place. La démarche est encore plus probante quand on reconnait la validité d'une forme actuellement non normée sous la plume de grands auteurs. C'est le cas du verbe obstiner de cet énoncé relevé sur un forum «on t'obstine pas que ça marche pas " dont la construction non pronominale est repérable au $\mathrm{xVII}^{\mathrm{e}}$ chez P. Corneille (1639): "Tout vostre discours, par un contraire effet, l'obstine en ses amours $»^{9}$. Un tel exemple permet, de fait, de situer les emplois sans les dévaloriser. La conscience de ces variantes chez les enseignantes permet de relativiser l'erreur des élèves et de considérer leur production comme un possible de la langue.

De même, mettre en parallèle ces réalisations très fréquentes en français : T'a pris le train? ou c'est lui qu'est venu non seulement avec leur forme normée mais aussi avec l'élision j'ai pris, c'est lui que tu vois permet de présenter ces formes dans leur logique, comme un possible de la langue qui n'est pas utilisé par la norme.

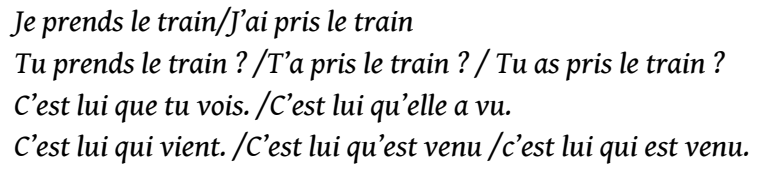

En conduisant à l'assimilation de qui et de $q u$ ', ces variantes sociologiques peuvent empêcher littéralement de comprendre la spécificité des relatifs si elles ne sont pas analysées. Or, de telles variantes, très courantes à l'oral, sont occultées en classe de français d'autant plus que la prise en compte du phénomène des variantes grammaticales devant initiale vocalique ${ }^{10}$ en langue standard est, elle-même, très limitée. Cependant ce phénomène ne peut être éludé en FLES sans conduire à des erreurs multiples : ma pareil-photo / Est-ce qu'il y a d'invités?/ Cet l'exercice/ S'elle veut / la petite vabo/ les\#enfants.... Or, si l'usage permet aux francophones, dans une certaine mesure, d'éviter ces erreurs de production, il reste que l'occultation de ces phénomènes est susceptible de perturber l'appréhension de la langue. Pour en donner un exemple, les étudiants à qui on n'a pas présenté le phénomène des variantes devant initiale vocalique pensent parfois que dans mon histoire, le nom histoire a changé de genre par rapport à une histoire. Resituer une variante sociologique comme t'a pris le 
train ou c'est lui qu'est venu parmi les variantes linguistiques standard permettrait donc de montrer les différentes logiques à l'œuvre dans ce phénomène lié à l'environnement vocalique et en déconstruisant certaines analyses faussées, de résoudre des conflits cognitifs larvés.

L'enseignante trouve alors la bonne posture devant une réalisation qu'il ne peut pas ignorer et qu'il ne doit en définitive ni légitimer dans un usage scolaire ni occulter. Mais la réflexion sur la prise en compte de la langue réelle de l'élève concerne aussi des éléments de la langue standard, comme le démontrent les études d'E. Guérin (2011) et de C. Péret et J.-P. Sautot (2018).

\section{Conclusion}

En définitive, au-delà de pratiques innovantes de la didactique du FLES, il apparait que des concepts qui lui sont propres et leurs implications pourraient innerver une réflexion sur la didactique du français. Ces concepts, comme la prise en compte de l'usage non partagé de la langue enseignée, la déconstruction de la langue, la fossilisation et l'anticipation des erreurs sont susceptible de donner toute leur portée à des concepts didactiques déjà existants en français langue première comme la progression, l'activité réflexive sur la langue et, in fine, de modifier les postures enseignantes. Dès lors, la question désormais rituelle de la formation des enseignants s'enrichit d'une nouvelle perspective : serait-il utile de former les futures enseignantes de français à la didactique de la langue FLES pour le bénéfice de tous les élèves ? Certes, le Capes de Lettres propose actuellement une option FLES mais cette option ne touche qu'une minorité de candidats. Attendre de tous les enseignantes de français la capacité de mettre à distance le présupposé selon lequel la langue enseignée serait partagée par les élèves, grâce à une formation en FLES en amont et en aval du Capes, aurait des impacts non seulement sur l'accueil des élèves allophones mais aussi sur la posture des enseignants de français et in fine sur l'efficacité de l'enseignement de la langue.

\section{BIBLIOGRAPHIE}

AUGER, N. (2005). Comparons nos langues : démarche d'apprentissage du français auprès d'enfants nouvellement arrivés (ENA) [DVD]. Montpellier : Scérén.

AVEZARD-Roger, C. \& Gourdet, P. (2017). « Présentation. Nouveaux Programmes et étude de la langue ». Le Français aujourd'hui 198, p. 5-12. En ligne : https://doi.org/10.3917/lfa.198.0005.

BEAUMANOIR-SECQ, M. (2018). Conceptualiser les classes de mots. À la recherche d'une grammaire utile aux élèves, dans la continuité et la cohérence. Bruxelles : Peter Lang.

Boulton, A. \& Tyne, H. (2014). Des documents authentiques aux corpus : démarches pour l'apprentissage des langues. Paris : Didier. 
BOUTET, J. (2002). « “I parlent pas comme nous”. Pratiques langagières des élèves et pratiques langagières scolaires ». Ville-École-Intégration Enjeux 130, p. 163-177.

BRISSAUD, C., CHEVROT, J.-P. \& LEFRANÇOIS, P. (2006). « Les formes verbales homophones en /E/ entre 8 et 15 ans : contraintes et conflits dans la construction des savoirs sur une difficulté orthographique majeure du français ». Langue Française 151, p. 74-93. En ligne : https://doi.org/ 10.3917/lf.151.0074.

BRISSAUD, C. \& COGIS, D. (2011). Comment enseigner l'orthographe aujourd'hui ? Paris : Hatier.

CHARTRAND, S.-G. (dir.) (2016). Mieux enseigner la grammaire. Pistes didactiques et activités pour la classe. Montréal : ERPI.

BYBEE, J. (1985). Morphology; A study of the relation between meaning and form, Amsterdam : John Benjamins.

BYBEE, J. \& MCCLELLAND, J. (2005). « Alternatives to the combinatorial paradigm of linguistic theory based on domain general principles of human cognition ». The Linguistic Review 22 (2-4),

p. 381-410. En ligne : https://www.researchgate.net/publication/

237442900_Alternatives_to_the_combinatorial_paradigm_of_linguistic_theory_based_on_general_principles_of_human_cognition.

CAMUSSI-NI, M.-A. (2020). « Le mot. Critique théorique et implications didactiques ». Tétralogiques

25. En ligne : http://www.tetralogiques.fr/spip.php?article160.

CAMUSSI-NI, M.-A. \& COATÉVAL, A. (2013). Comprendre la grammaire. Une grammaire à l'épreuve de la didactique du FLE. Grenoble : Presses universitaires de Grenoble.

CAMUSSI-NI, M.-A., COATÉVAL, A. \& FOLMER, C. (2016). « Élèves FLS, Élèves FLM : L'hétérogénéité, tremplin à un apprentissage réfléchi de la langue ». In : A. Pégaz Paquet \& L. Cadet (éds), Les Langues à l'école, la langue de l'école. Arras: Presses universitaires d'Artois, p. 115-128.

CAMUSSI-NI, M.-A., COATÉVAL, A. \& THUILIER, J. (2016). « Exploiter un travail de corpus sur la place de l'épithète pour élaborer une progression didactique en FLE ». Linguistik online 78. En ligne : https://doi.org/10.13092/lo.78.2949.

CHISS, J.-L. (1997). «Éléments de problématisation pour l'enseignement/apprentissage du français aux élèves « non francophones ». In : D. Boyzon-Fradet \& J.-L. Chiss (dirs), Enseigner en classes hétérogènes, Ecole et immigration. Paris : Nathan. Accessible sur le lien. http://casnav.ac-creteil.fr/ IMG/pdf/chiss.pdf.

COGIS, D. (2001). « Difficultés en orthographe. Un indispensable réexamen ». Revue française de linguistique appliquée, VI-I, p. 47-61. En ligne : https://doi.org/10.3917/rfla.061.0047.

COGIS, D. (2004). « Une approche de la morphographie. L'exemple d'une séquence sur l'accord de l'adjectif ». Lidil 30, p. 73-86. En ligne : https://doi.org/10.4000/lidil.743.

COGIS, D. (2005). Pour enseigner et apprendre l'orthographe. Nouveaux enjeux, pratiques nouvelles, école, collège. Paris : Delagrave.

DAVID, J. \& RENVOISÉ, C. (2010). « La morphologie verbale, repérer les complexités et les régularités », Synergies France 6, p. 61-75. En ligne : https://gerflint.fr/Base/France6/ france6.html.

DEWAELE, J.-M. (2003). « Compte rendu - hommage : l'œuvre de L. Selinker ». Linx 49, p. 153-159. En ligne : https://doi.org/10.4000/linx.564. 
ÉLALOUF, M.-L. (2005). « De la 6e à la 1re, comment mobilisent-ils leurs connaissances sur la langue dans des tâches d'explication? » Pratiques 125-126, p. 157-178. En ligne : https://doi.org/10.3406/ prati.2005.2065.

ÉLALOUf, M.-L., Cogis, D. \& GouRdet, P. (2011). « Maitrise de la langue à l'école et au collège. Progressions et contradictions dans les programmes de 2008. ». Le Français aujourd'hui 173, p. 33-44. En ligne : https://doi.org/10.3917/lfa.173.0033.

FOURNIER, N. (2002), Grammaire du français classique, Paris : Belin.

GOURDET, P. \& ROUBAUD, M.-N. (2016). « L'enseignement du verbe à l'école. Des tensions entre enseignants et élèves de CM2 ». Pratiques 169-170. En ligne : http://journals.openedition.org/ pratiques/3059.

GUÉRIN, E. (2010). « L'“outre-langue” des enseignants ou le mythe d'une langue mono-variétale ». Pratiques 145-160, p. 45-54. En ligne : https://doi.org/10.4000/pratiques.1501.

GUÉRIN, E. (2011). « La variation de la langue dans les manuels scolaires du cycle 3 et du collège ». Le Français aujourd'hui 173, p. 57-70. En ligne : https://doi.org/10.3917/lfa.173.0057.

LAPARRA, M. (2003). «Variations et usages linguistiques dans et hors l'école ». Le Français aujourd'hui 143, p. 7-16. En ligne : https://doi.org/10.3917/lfa.143.0007.

LARGY, P., COUSIN, M.-P. \& FAYOL, M. (2004). « Acquérir le pluriel des noms. Existe-t-il un effet de fréquence du nom? ». Lidil 30, 39-54. En ligne : https://doi.org/10.4000/lidil.663.

LE LEVIER, H., BRISSAUD, C. \& HUARD, C. (2018). « Le raisonnement orthographique chez des élèves de troisième : analyse d'un corpus d'entretiens métagraphiques ». Pratiques 177-178, En ligne : http://journals.openedition.org/pratiques/4464.

PÉRET, C. \& SAUTOT, J.-P. (2018). « Le futur à l'école - tensions entre situation dans le temps et morphologie verbale. Les programmes scolaires, une matrice d'exclusion?». Linx 77 En ligne : http://journals.openedition.org/linx/2734.

SELINKER, L. (1972). « Interlanguage ». International Review of Applied Linguistics 10, p. 219-231.

SELINKER, L. \& Lakshamanan, U. (1992). « Language transfer and fossilization : The Multiple Effects Principle ». In : Selinker L. \& Gass S. (éds). Language Transfer In Language Learning. Amsterdam : John Benjamins, p. 197-216.

TISSET, C. (2010), Enseigner la langue française, la grammaire, l'orthographe et la conjugaison à l'école. Paris : Hachette.

TOTEREAU, C., Barrouillet, P. \& Fayol M. (1998). « Overgenralizations of number inflections in the learning of written french. The case of nouns and verbs ». British journal of Developmental Psychology 16, p. 447-464.

TRÉVISE, A. (1997), « Métalexique, métadiscours et interactions métalinguistiques », Linx 36, p. 41-54. En ligne : https://doi.org/10.3406/linx.1997.1452.

VARGAS, C. (2011). « La maitrise de la langue. Quand les programmes cesseront de tourner en rond ». Le Français aujourd'hui 173, p. 113-117. En ligne : https://doi.org/10.3917/lfa.173.0113.

VIGNER, G. (2001). Enseigner le français comme langue seconde. Paris : Éd. Clé International.

VOGEL, K. (1995). L'Interlangue. La langue de l'apprenant. Toulouse : Presses universitaires du Mirail. 


\section{Manuels :}

Beltrando, B. (dir.) (2019). Français $2^{\text {de }}$. Motifs littéraires. Paris : Didier.

Huchet C \& Bastier H. (2019) [2017]. Mandarine CM2, cycle 3. Français. Paris : Hatier.

Damas X. (dir.) (2019). Langue Française et Expression $2^{\text {de }}$. Paris : Hatier.

\section{NOTES}

1. L'expression «Français langue première " équivalente à «Français langue maternelle » sera préférée ici et parfois dénommée par le sigle FLP.

2. L'astérisque placé avant un terme, indique l'agrammaticalité de l'énoncé, dans cet article.

3. De nombreux chercheurs en didactique du FLM soulignent ce décalage des Instructions Officielles françaises et préconisent une progression mieux adaptée (par exemple, Élalouf, Cogis \& Gourdet, 2011 ; Vargas, 2011).

4. Voir à ce sujet Camussi-Ni (2020).

5. Voir les listes réalisées à partir du classement des mots en fréquence de Brunet sur le site Eduscol.

6. Sur les travaux de L. Selinker, on peut lire Dewaele (2003). Voir aussi, sur le sujet de l'interlangue, Vogel (1995).

7. Voir par exemple, le blog Enseigner la langue de l'auteure, sur Wordpress, qui diffuse ce type de corpus de découverte créés par des étudiants de Meef Lettres.

8. Ces exemples sont extraits de La Grammaire du français classique de N. Fournier (2002) qui répertorie une liste fournie de ces verbes.

9. Occurrence repérée (parmi quelques autres de la même époque) grâce à la banque de données Frantext.

10. Pour une présentation unifiée du phénomène, voir Comprendre la grammaire (Camussi-Ni \& Coatéval, 2013)

\section{RÉSUMÉS}

Et si se former à l'enseignement du français langue étrangère, parce que cela signifie apprendre nécessairement à prendre en compte l'usage non partagé de la langue enseignée, permettait d'acquérir une meilleure posture en tant que professeur de français ? Dans cet article, nous interrogeons ce postulat paradoxal à l'aide d'éléments comparés de recherches en didactiques du français langue première et $d u$ français langue étrangère et seconde. Nous montrons ses répercussions possibles en termes de prise en compte des usages réels des élèves sur le plan sociolinguistique; de la nécessaire déconstruction de la langue étudiée à l'aide de corpus ; mais 
aussi en termes de vigilance sur l'acquisition supposée du métalangage et sur d'éventuelles fossilisations au cours de l'apprentissage.

What if learning how to teach French as a foreign language, which necessarily means learning reckoning that the use of the language taught is not obviously shared, made it possible to acquire a better posture as a teacher of French? We question this paradoxical assumption using comparative elements of research from the didactics of French as a first language and of French as a foreign and second language. We show its possible impacts when observing the real uses of students at the sociolinguistic level; the necessary deconstruction of the language studied using corpora and the need to be aware of the supposed acquisition of metalanguage and of possible fossilizations during learning.

\section{INDEX}

Keywords : Interdidacticity, French as a first language, French as a foreign and second language, fossilization, grammar, anticipation of errors.

Mots-clés : Interdidacticité, Français langue première, FLES, fossilisation, grammaire, anticipation des erreurs

\section{AUTEUR}

\section{MARIE-ARMELLE CAMUSSI-NI}

Université de Rennes, Cellam, 35000 Rennes, France 